\title{
Estudo Preliminar do Uso de Meta-heurísticas para Seleção de Canais em Sistemas BCI-SSVEP*
}

\author{
Raquel Queiroz $^{1}$, Denis G. Fantinato ${ }^{1}$ \\ ${ }^{1}$ Centro de Matemática, Computação e Cognição \\ Universidade Federal do ABC (UFABC) - Santo André - SP - Brazil \\ \{raquel.queiroz, denis.fantinato\}@ufabc.edu.br
}

\begin{abstract}
Classification of electroencephalography (EEG) signals is a crucial problem in Brain-Computer Interfaces (BCI) systems. The EEG signals can be seen as a set of temporal series, being the use of Recurrent Neural Networks (RNNs) interesting for their processing, particularly the Long Short-Term Memory (LSTM) network. In this work, we propose the use of a LSTM classifier along with the Genetic Algorithm (GA) or the Clonal Selection Algorithm (CSA) for selection of electrodes in a set of artificial EEG data.
\end{abstract}

Resumo. A classificação dos sinais eletroencefalográficos (EEG) é um problema fundamental nos sistemas de Interface Cérebro-Computador (BCI). Os sinais de EEG podem ser vistos como um conjunto de séries temporais, sendo interessante o uso das Redes Neurais Recorrentes (RNNs) para seu processamento, em particular a rede LSTM (Long Short-Term Memory). Neste artigo é proposto uma estrutura de classificação baseada em LSTM utilizando o Algoritmo Genético (GA) e o Algoritmo de Seleção Clonal (CSA) para realizar a seleção do número de canais em um conjunto de dados de EEG artificiais.

\section{Introdução}

Um sistema de Interface Cérebro-Computador (BCI, do inglês Brain-Computer Interface) é um sistema capaz de traduzir padrões de ativações cerebrais e ativar comandos no mundo exterior [Bablani et al. 2019]. Esse sistema inicialmente faz a aquisição do sinal cerebral, geralmente sinais de eletroencefalogramas (EEG), uma técnica não invasiva e de custo relativamente baixo, porém apresenta baixa resolução espacial e um alto nível de ruído, o que torna difícil extrair informações úteis [Nam et al. 2018]. Após a captura, as informações são processadas nas etapas de pré-processamento, extração e seleção de atributos e, por fim, a classificação, cujo resultado é utilizado para acionar comandos de controle em dispositivos protéticos, cadeiras de rodas ou computadores.

Dentre os algoritmos de classificação, as Redes Neurais Artificiais (ANNs, do inglês Artificial Neural Networks) são inspiradas em sistemas neurais biológicos com recursos como computação paralela, não linearidade, adaptabilidade, capacidade de resposta e tolerância a falhas [Goodfellow et al. 2016]. Uma classe de ANN que merece destaque é a Rede Neural Recorrente (RNN, do inglês Recurrent Neural Networks). Particularmente, destacando a rede Long Short-Term Memory (LSTM) foi criada para ser

\footnotetext{
*Esse trabalho foi parcialmente financiado pela Fundação de Amparo à Pesquisa do Estado de São Paulo (FAPESP), processo no 2020/10014-2.
} 
capaz de processar informações de curto e longo prazo. Por tratar os dados de entrada como séries temporais, torna-se uma ferramenta promissora para o tratamento de sinais de EEG. Em um sistema BCI, a etapa de classificação pode ser auxiliada pela etapa de seleção de canais (dos eletrodos), usando apenas aqueles mais relevantes para a tarefa [Nam et al. 2018]. Apesar de contar apenas com algumas dezenas de eletrodos, as combinações possíveis para seleção geram um espaço de busca consideravelmente grande, muitas vezes inviabilizando a busca exaustiva. Assim, será analisado neste trabalho o uso de duas meta-heurísticas para esta busca: o Algoritmo Genético (GA, do inglês Genetic Algorithm) e o Algoritmo de Seleção Clonal (CSA, do inglês Clonal Selection Algorithm). Serão considerados dados artificiais de EEG para a análise do sistema BCI.

\section{As Interfaces Cérebro-Computador}

Em um sistema BCI, existe uma ampla gama de técnicas comumente empregadas em cada uma das etapas, e a escolha do conjunto de técnicas a ser adotado depende do paradigma escolhido [Nam et al. 2018]. Neste trabalho, será dado foco ao paradigma de BCI baseado em Potencial Evocado Visual em Regime Estacionário (SSVEP, do inglês Steady-State Visual Evoked Potential), em que utilizam-se estímulos visuais que oscilam em frequências específicas. Nesse caso, métodos seletores de bandas e de análise espectral (Transformada de Fourier, de Welch e Wavelet) [Nam et al. 2018] podem ser utilizados. No entanto, recentes métodos de aprendizado profundo (DL, do inglês Deep Learning) aplicados a BCI demonstraram altas taxas de acurácia, mesmo sem as etapas de pré-processamento e extração de atributos [Nam et al. 2018]. Assim, neste trabalho, será gerado dados artificiais de EEG e analisado um sistema BCI composto apenas da etapa de seleção de canais (usando meta-heurísticas) e da etapa de classificação (com a LSTM).

\subsection{Conjunto de Dados baseados em Potencial Evocado}

Em SSVEP, o estímulo visual gera a resposta nos sinais de EEG com conteúdo em mesma frequência do estímulo. Assim, uma maneira de gerar artificialmente os dados de sinais EEG-SSVEP é combinar senoides na frequência de estímulo a ruídos inerentes do processo. Neste trabalho, os sinais dos eletrodos foram gerados como:

$$
s_{i}(n)=\cos \left(2 \pi f_{j} t n / T\right)+\eta(i),
$$

em que $i=\{1, \ldots, 16\}$ é o índice do eletrodo, $f_{j}$ é a frequência de estímulo, aqui podendo ser $10,15,20$ e $25 \mathrm{~Hz}, n=\{0, \ldots, 255\}$ e período $T=256 \mathrm{~Hz}$. Foram geradas 200 instâncias para cada frequência de estímulo. Também foi inserido ruído branco gaussiano $\eta$, com média nula e variância igual a $i$. Assim, à medida que se aumentam os índices do canal, mais ruído é adicionado. Ressaltando que esse tipo de ruído não acontece na prática, sendo portanto utilizados para um estudo teórico da seleção de canais pelas metaheurísticas, em que, espera-se que seja capaz de selecionar os canais com menor índice.

\subsection{Seleção de Canais Usando Meta-heurísticas}

Os GAs são uma técnica de busca probabilística global inspirada na evolução darwiniana e visam encontrar soluções ótimas por meio da simulação de um processo evolutivo natural. Os operadores genéticos como crossover (feita por Single Point), mutação (aqui, Bit Flip) e seleção (feita por torneio) são usados para manter a diversidade genética, que é necessária para o processo de evolução [Bouktif et al. 2020]. Já o CSA é um modelo 
inspirado na teoria de seleção clonal (baseado no sistema imunológico). Seu princípio de funcionamento usa a resposta imune no corpo chamada de anticorpo, que é induzida quando uma substância estranha chamada antígeno é detectada. Consiste em quatro componentes que são seleção (feita por elitismo), expansão clonal, hipermutação e reseleção [Pang et al. 2015]. O CSA possui similaridade com o GA, porém pode alcançar um conjunto diversificado de soluções ótimas locais, enquanto todas as soluções candidatas no GA convergem para a melhor solução encontrada.

No contexto de BCI, o GA e o CSA deverão encontrar quais canais de eletrodos são mais úteis para o treinamento da rede neural LSTM, em termos de maior taxa de acerto, ou seja, em canais cujo fitness (acurácia do modelo) sejam mais altos. Para ambas as meta-heurísticas, uma solução candidata é um vetor com 16 bits, cada um associado a um eletrodo. Um bit 1 na $i$-ésima posição indica que o sinal do eletrodo $i$ será usado na classificação.

\subsection{Classificador Baseado em Redes Neurais Recorrentes}

Os sinais dos eletrodos selecionados são então submetidos ao classificador, que é uma rede LSTM. Esta rede possui estrutura fixa, sendo 3 camadas LSTM e uma camada densa, conforme apresentado na Tab. 1. sendo $b$ o batchsize, igual a 402.

\begin{tabular}{ccc}
\hline Camada (tipo) & Dim. Saída & N. Parâmetros \\
\hline LSTM & $(b, 256,10)$ & 1080 \\
LSTM & $(b, 256,5)$ & 320 \\
LSTM & $(b, 3)$ & 108 \\
Dense & $(b, 4)$ & 20 \\
\hline
\end{tabular}

Tabela 1. Estrutura do classificador baseado em LSTM

A saída da camada Dense (com função de ativação softmax) tem dimensão 4, cada uma correspondendo a uma determinada classe (frequências do estímulo).

\section{Resultados}

Baseado no conjunto de dados baseados em Potencial Evocado, para cada experimento foram criadas amostras a partir de 16 canais e 4 rótulos de frequência em [10, 15, 20, 25] Hz. Foram utilizadas 600 amostras - organizadas como $(600,256,16)$, onde são (amostras, experimentos, eletrodos) -, das quais 402 amostras foram para o treino do modelo e 198 para teste. O modelo treinado com todos os canais sem nenhuma metaheurística aplicada obteve uma acurácia média de $26.8 \%$, com o desvio padrão de $1.5 \%$ para 30 repetições, em que cada repetição foi considerado o modelo com maior precisão dentre 4 modelos treinados.

Utilizando a meta-heurística de GA, foi executado para um total de 100 gerações, a quantidade 60 indivíduos e o percentual de mutação em 10\%. A Fig. 1a exibe a precisão ao longo das geração usando o GA. A melhor precisão ocorreu primeiramente na geração 46 com $96.5 \%$ utilizando os canais $[0,1,2,4,5,6]$, sendo encontrada novamente a partir da geração 95 .

Em contrapartida com o CSA, Fig. 1b, utilizando a geração de amostras com os mesmos parâmetros anteriormente descritos, o experimento com a maior precisão foi de 


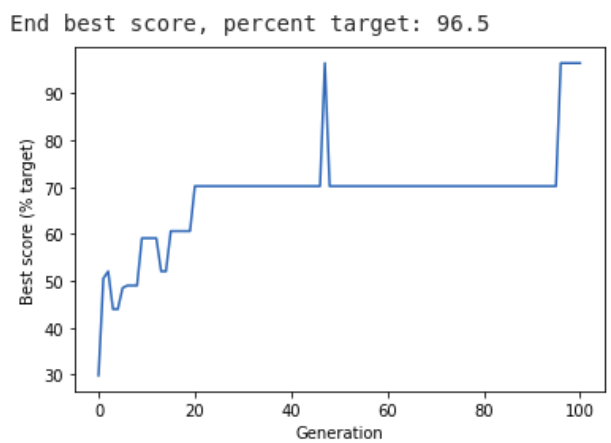

(a) GA

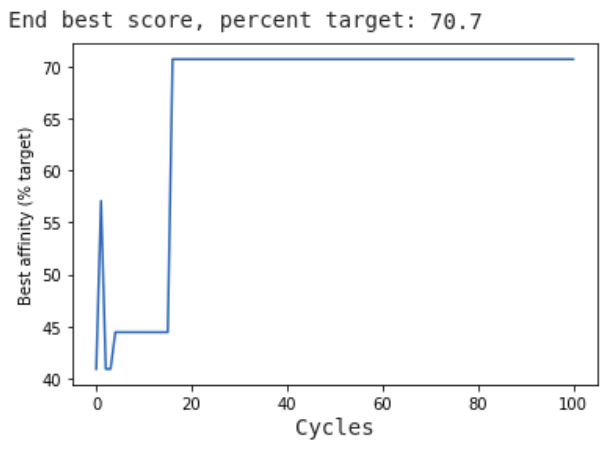

(b) CSA

Figura 1. Taxa de acerto ao longo das gerações usando (a) GA e (b) CSA.

$70.7 \%$ na iteração 15 . Os canais desta solução foram $[4,12,13,14]$ e o desvio padrão do modelo treinado com estes canais e com 30 repetições foi de $2.8 \%$.

\section{Conclusão}

Neste trabalho foi proposto o uso de meta-heurísticas para classificar sinais de EEG artificiais (baseados em Potenciais Evocados) e, como mostrado na seção de resultados, tanto o GA quanto o CSA conseguiram encontrar soluções com baixa complexidade estrutural e melhor precisão no treino de LSTMs.

A partir dos experimentos, é possível observar que os canais a serem escolhidos pelo GA são os primeiros canais, que revela uma escolha coerente com os sinais gerados artificialmente. Assim, o GA tende a remover canais cujo a quantidade de ruído seja mais elevada. Em alternativa, os experimentos com o algoritmo CSA obtiveram uma precisão menor do que com o GA, selecionando os canais 4, 12, 13 e 14. Muito provavelmente, isto decorre do fato do algoritmo de seleção clonal alcançar um conjunto mais diversificado de soluções ótimas locais, enquanto todas as soluções candidatas no GA convergem para a melhor solução encontrada. Posteriormente, a intenção seria evoluir o estudo aplicando as meta-heurísticas em sinais de EEG reais baseados em SSVEP.

\section{Referências}

Bablani, A., Edla, D., Tripathi, D., and Cheruku, R. (2019). Survey on brain-computer interface: An emerging computational intelligence paradigm. ACM Computing Surveys, 52(1):1-32.

Bouktif, S., Fiaz, A., Ouni, A., and Serhani, M. A. (2020). Multi-sequence lstm-rnn deep learning and metaheuristics for electric load forecasting. Energies, 13(2):391.

Goodfellow, I., Bengio, Y., Courville, A., and Bengio, Y. (2016). Deep Learning, volume 1. MIT press Cambridge.

Nam, C. S., Nijholt, A., and Lotte, F. (2018). Brain-Computer Interfaces Handbook: Technological and Theoretical Advances. CRC Press.

Pang, W., Wang, K., Wang, Y., Ou, G., Li, H., and Huang, L. (2015). Clonal selection algorithm for solving permutation optimisation problems: a case study of travelling salesman problem. In International Conference on Logistics Engineering, Management and Computer Science (LEMCS 2015), pages 575-580. Atlantis Press. 